\title{
Benzoyl Peroxide Formulated Polycarbophil/Carbopol 934P Hydrogel with Selective Antimicrobial Activity, Potentially Beneficial for Treatment and Prevention of Bacterial Vaginosis
}

\author{
Shiqi Xu, ${ }^{1}$ Veronica L. Cavera, ${ }^{2}$ Michael A. Rogers, ${ }^{1}$ Qingrong Huang, \\ Konstantin Zubovskiy, ${ }^{3}$ and Michael L. Chikindas ${ }^{1,4}$ \\ ${ }^{1}$ School of Environmental and Biological Sciences, Rutgers State University, 65 Dudley Road, New Brunswick, NJ 08901, USA \\ ${ }^{2}$ Department of Biochemistry and Microbiology, Rutgers State University, 76 Lipman Drive, New Brunswick, NJ 08901, USA \\ ${ }^{3}$ Scientelle, LLC, Morristown, NJ 07906, USA \\ ${ }^{4}$ Bioronus, LLC, Highland Park, NJ 08904, USA
}

Correspondence should be addressed to Qingrong Huang; qhuang@aesop.rutgers.edu and Michael L. Chikindas; tchikindas@aesop.rutgers.edu

Received 24 July 2013; Revised 20 September 2013; Accepted 2 October 2013

Academic Editor: Gregory T. Spear

Copyright (C) 2013 Shiqi Xu et al. This is an open access article distributed under the Creative Commons Attribution License, which permits unrestricted use, distribution, and reproduction in any medium, provided the original work is properly cited.

The human vagina is colonized by a variety of indigenous microflora; in healthy individuals the predominant bacterial genus is Lactobacillus while those with bacterial vaginosis (BV) carry a variety of anaerobic representatives of the phylum Actinobacteria. In this study, we evaluated the antimicrobial activity of benzoyl peroxide (BPO) encapsulated in a hydrogel against Gardnerella vaginalis, one of the causative agents of $\mathrm{BV}$, as well as indicating its safety for healthy human lactobacilli. Herein, it is shown that in well diffusion assays $G$. vaginalis is inhibited at $0.01 \%$ hydrogel-encapsulated BPO and that the tested Lactobacillus spp. can tolerate concentrations of BPO up to $2.5 \%$. In direct contact assays (cells grown in a liquid culture containing hydrogel with $1 \%$ BPO or BPO particles), we demonstrated that hydrogels loaded with $1 \%$ BPO caused 6-log reduction of G. vaginalis. Conversely, three of the tested Lactobacillus spp. were not inhibited while L. acidophilus growth was slightly delayed. The rheological properties of the hydrogel formulation were probed using oscillation frequency sweep, oscillation shear stress sweep, and shear rate sweep. This shows the gel to be suitable for vaginal application and that the encapsulation of BPO did not alter rheological properties.

\section{Introduction}

The healthy human vagina is colonized by a variety of bacterial species with lactobacilli being a predominant group of microorganisms. The cause and etiopathogenesis of bacterial vaginosis (BV) is still poorly understood; however, it has been extensively shown that when the natural ecology shifts to mainly Gram-negative Actinobacteria, microbial disease such as BV can occur [1]. BV affects one in three women in the United States. Initially, infection leads to discomfort and a foul discharge while long-term infection has been correlated with increased risk of pelvic inflammatory disease, sexually transmitted infections, and pregnancy complications including preterm birth $[2,3]$. Advanced methodological approaches utilizing qPCR and deep sequencing confirm BV as a multispecies infection $[4,5]$. However, G. vaginalis is observed in approximately $70 \%$ of tested women regardless of whether the individual is considered positive for BV based on the Nugent criteria [5-7].

Current Food and Drug Administration (FDA) approved treatments include nitroimidazoles (i.e., metronidazole) or the lincosamide clindamycin [8]. These antibiotics alter the indigenous microflora by inhibiting both the problematic and healthy bacterial species. G. vaginalis and other anaerobic bacteria grow in complex biofilms; therefore, a high dose of antibiotics is required to inhibit their growth. This high-dose requirement causes wide-spectrum microbial elimination and arrests the competitive exclusion capability of Lactobacillus leading to increased tolerance of BV-associated microorganisms [9]. Recurrence of resistant BV-associated 


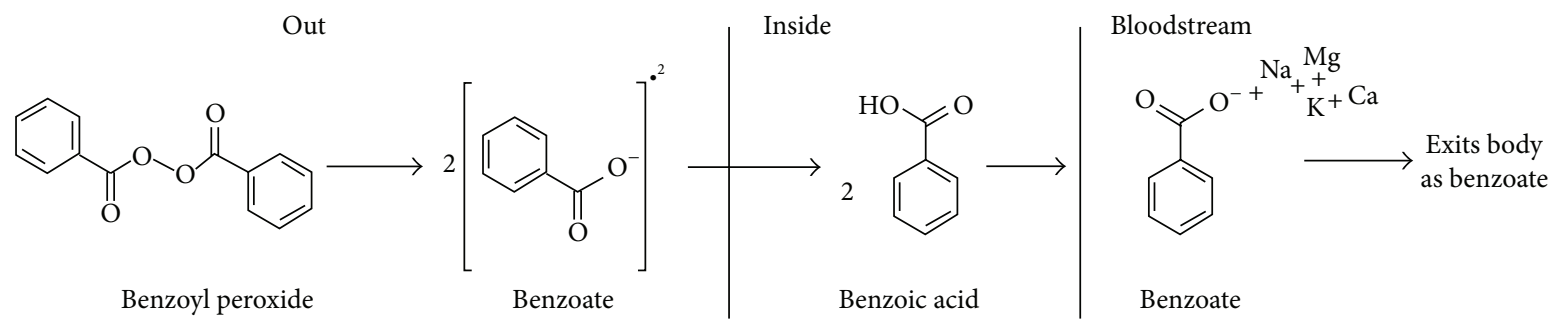

FIGURE 1: The breakdown of BPO upon contact with epithelium. BPO undergoes a homolytic fission resulting in two benzoate radicals which pass into the bloodstream where it is protonated into benzoic acid then deprotonated. Free cations in the blood will be attracted, but no further modifications will occur while in the body.

pathogens is seen in more than $50 \%$ of women up to a year following treatment from metronidazole or clindamycin $[10,11]$. Although there is no established causation between antibiotic usage and recurrence incidence, it is plausible that the lack of selectivity in available antibiotic treatment options may severely disrupt restoration of normal vaginal microbiota, which could prevent BV recurrence [8]. Lactobacilli are naturally occurring representatives of the vaginal microbiota, beneficiary to overall vaginal health. These bacteria effectively protect the vaginal environment against pathogens by producing natural antimicrobials such as hydrogen peroxide, bacteriocins, and weak organic acids $[9,12]$. Usually, healthy vaginal environments are colonized by predominantly one or two species of lactobacilli (generally L. crispatus or L. iners). Women with recurrent BV infections have been documented to have a wider variety of lactobacilli including but not limited to L. gasseri, L. plantarum, L. jensenii, and L. vaginalis $[13,14]$. Due to this wide variety of nonpathogenic bacteria, it is imperative to treat the pathogenic microorganisms with a more selective antimicrobial which is harmless to lactobacilli and does not promote resistance in pathogens.

$\mathrm{BPO}$ is an organic peroxide included on the World Health Organization (WHO) Model Lists of Essential Medicines $[15,16]$. It is identified as safe for human use and is found in a variety of products ranging from flour bleaching agents to cleaning products. Further, BPO is often used in topical formulations because it is reasonably stable in gel solutions. Free radical generation from $\mathrm{BPO}$ has not been associated with acquired resistance in acne-associated cases [16, 17]. Resistance from BPO usage has not been reported for G. vaginalis; moreover, naturally occurring Lactobacillus spp. produce hydrogen peroxide which also utilizes a homolytic fission to competitively inhibit other bacteria. When used in topical medicinal or cosmetic formulations, such as in a hydrogel treating acne, BPO undergoes homolytic fission resulting in two benzoate radicals due to the perester nature of the compound. As benzoate passes through the epithelium, a proton is acquired, altering the compound into benzoic acid which diffuses freely into the bloodstream where it is deprotonated due to its low pKa [18]. Free cations in the blood will be attracted to benzoate and the resultant structure is excreted without further alteration (Figure 1).

Antibiotics targeted at vaginal pathogens are administered either orally or intravaginally. The latter requires a delivery system which provides physical contact of the antimicrobial agent with the mucosa. Gels represent one of the most frequently used intravaginal delivery systems due to their acceptability, feasibility, and low cost [19]. This delivery system reduces the bacterial load through the formation of a physical barrier with the vaginal topography which enhances natural defenses of the vaginal environment $[12,20]$. The most effective gels also need to be mucoadhesive; this allows for an extended period of contact between the encapsulated drug and the vaginal epithelium while maintaining its properties $[21,22]$.

Several currently available vaginal hydrogels (Crinone, Replens, RepHresh, Advantage-S, Miphil, etc.) are utilizing bioadhesive properties of polycarbophil which belongs to the poly(acrylic) acid group.

The underlying hypothesis for this study was that the BPO encapsulated in bioadhesive hydrogel would have a selective antibacterial profile required for a sustained treatment effect in BV, making it a good candidate for further development. In this study, we developed a polycarbophil/Carbopol 934P hydrogel with encapsulated BPO. The resulted gel formulation was capable of controlling the BV-associated pathogen $G$. vaginalis while not inhibiting four healthy vaginal microorganisms (L. acidophilus, L. gasseri, L. plantarum, and L. vaginalis). This gel is conceptually close to already marketed products with proven safety features, mimics the function of the peroxide-producing lactobacilli, and therefore could be a promising candidate for treatment of BV. The rheological properties of the formulation evaluated in vitro indicate that the gel will maintain its structure within the vaginal environment.

\section{Materials and Methods}

2.1. Chemical Compounds. Noveon AA-1 polycarbophil was obtained from Lubrizol Advanced Materials, Inc. (Cleveland, OH, USA). Hydrous BPO (74\% BPO, 26\% water), Carbopol 934P, acetic acid, and sodium acetate were from Spectrum Chemical Manufacturing Corp. (New Brunswick, NJ, USA).

Vaginal fluid simulant (VFS) used in rheological evaluation was composed of $\left(\mathrm{g} / \mathrm{L} \mathrm{ddH}_{2} \mathrm{O}\right): 3.51$ sodium chloride, 0.222 calcium hydroxide, 3.41 potassium hydroxide, 0.018 bovine serum albumin, 2.0 lactic acid, 1.0 acetic acid, 0.16 glycerol, 0.4 urea, and 5.0 glucose. To adjust $\mathrm{pH}$ to $4.2,6 \mathrm{~N}$ hydrochloric acid was added [23]. Sodium chloride, bovine serum albumin, lactic acid, and glucose were purchased from 
Sigma-Aldrich Co (St. Louis, MO, USA). Calcium hydroxide, glycerol, and urea were purchased from Fisher Scientific Inc, (Pittsburgh, PA, USA). Potassium hydroxide and hydrochloric acid were purchased from VWR International LLC (Radnor, PA, USA). Clindamycin (Tokyo Chemical Industry, Tokyo, Japan) was used as a positive control for well diffusion experiments. Aqueous stock solutions of clindamycin were filter-sterilized through $0.2 \mu \mathrm{m}$ syringe filters (NALGENE, Rochester, NY, USA).

2.2. Bacterial Strains, Growth, and Conditions. G. vaginalis ATCC 14018 was used as the BV-associated pathogen and was stored at $-80^{\circ} \mathrm{C}$ in Brain Heart Infusion (BHI) medium (Difco, Sparks, MD, USA) supplemented with 3\% horse serum (HS) (JRH Biosciences, KS, USA) and 15\% (by volume) glycerol. Frozen stocks were cultured on human blood bilayer Tween (HBT) agar (Remel, Lenexa, KS, USA) and grown at $37^{\circ} \mathrm{C}$ in $5 \% \mathrm{CO}_{2}$ and $2.5 \% \mathrm{H}_{2}$ for 48 hours using EZ Anaerobe Container System GasPak (Becton, Dickinson and Co., Sparks, MD, USA). Experiments were performed in Type A Coy Laboratory Vinyl Anaerobic Chamber (Coy Laboratory Products, Grass Lake, MI, USA). The anaerobic conditions are identical to those provided by the EZ Anaerobe Single colonies which were streaked onto HBT plates for the modified agar streak well diffusion assay. All media and agar for $G$. vaginalis were preincubated in the aforementioned anaerobic conditions for 24 hours to remove oxygen-related stress.

L. vaginalis ATCC 49540, L. gasseri ATCC 33323, L. plantarum ATCC 39268, and L. acidophilus ATCC 4356 were representative of the normal flora of a healthy vagina $[13,14]$. L. gasseri and L. vaginalis are representative of vaginal flora of healthy women while L. acidophilus and L. plantarum have been isolated in women with recurrent BV infections. These were selected as they represent a wider net of nonpathogenic bacteria that should not be inhibited to ensure continued health and maintenance of the vaginal environment. They were stored at $-80^{\circ} \mathrm{C}$ in DeMan, Rogosa, and Sharpe (MRS) broth (Oxoid, Hampshire, England) containing 15\% glycerol by volume. The cells were plated on $1.5 \% \mathrm{w} / \mathrm{v}$ MRS agar and grown aerobically at $37^{\circ} \mathrm{C}$. For experimental procedures, single colonies were inoculated in $20 \mathrm{~mL}$ of MRS and grown aerobically for 24 hours with agitation (100 RPM). Cells were subcultured twice before use. For all experiments, $200 \mu \mathrm{L}$ of the overnight culture was transferred into $20 \mathrm{~mL}$ of fresh broth.

2.3. Hydrogel Preparation. Base gel was defined as hydrogel without BPO. The base gel is prepared as follows $(\mathrm{w} / \mathrm{w})$ : polycarbophil/Carbopol 934P were hydrated in double distilled water. Sodium acetate, acetic acid, Carbopol gel, and glycerol were slowly added to polycarbophil gel while stirring. The final concentrations of these components were $2 \%$ polycarbophil, 1\% Carbopol 934P, 15\% glycerol, 0.049\% sodium acetate, and $0.038 \%$ acetic acid. To elevate $\mathrm{pH}$ of base gel from 3.20 to $4.50,5 \mathrm{M}$ sodium hydroxide solution was used.

BPO particles were evenly dispersed in base gel at predetermined concentrations from $0.01 \%$ to $10 \%$ in Nasco Whirl-Pak bags (Fisher Scientific Inc., Pittsburgh, PA, USA). The gel was then placed into $50 \mathrm{~mL}$ tubes (Becton Dickinson,
Franklin Lakes, NJ, USA) and centrifuged at 720 relative centrifugal force (RCF in $g$ forces) at ambient temperature for 10 minutes in an Allegra 21R Centrifuge (54180 fixed angle rotor) (Analytical Instrument Brokers, LLC, MN, USA).

2.4. Agar Streak and Soft Agar Overlay Well Diffusion Assays. A modified agar streak method described by Waksman and Reilly was used for G. vaginalis as the microorganism does not grow well in soft agar [24]. Briefly, colonies isolated from frozen stock were restreaked on preincubated (i.e., kept in the anaerobic environment overnight) HBT plates. To obtain a homogenous lawn of Lactobacillus spp., $5 \mathrm{~mL}$ of MRS soft $(0.7 \% \mathrm{w} / \mathrm{v})$ agar was inoculated with overnight cultures $\left(10^{7}\right.$ $\mathrm{CFU} / \mathrm{mL})$ and evenly distributed over MRS (1.5\% w/v) agar plate. Soft agar overlay plates were dried for approximately one hour in a Purified Class II Safety Cabinet (LabConco Co., Kansas City, MI, USA).

Wells were then aseptically punched through the soft agar overlay and the hard agar with the back of a $1000 \mu \mathrm{L}$ tip. Each well was filled with $100 \mu \mathrm{L}$ of gel formulation. Fifty $\mu \mathrm{L}$ of $100 \mu \mathrm{g} / \mathrm{mL}$ clindamycin was used as a positive control. Plates were incubated in an anaerobic jar (Sigma Aldrich, St. Louis, MO, USA) for 24 hours at $37^{\circ} \mathrm{C}$. After incubation, zones of inhibition were measured with Vernier calipers (Nova-Tech, Houston, TX, USA). This experiment was performed at least two times in triplicates.

2.5. Direct Contact Inhibition Studies. Inhibition studies were conducted using the time kill method as described by Liang et al. [25] with modifications. All bacteria were grown in contact with $5 \mathrm{~mL} \mathrm{1 \%}$ BPO gel, $5 \mathrm{~mL}$ base gel, or $0.05 \mathrm{~g}$ BPO particles (0.067 $\mathrm{g}$ hydrous BPO). BPO particles are defined as the hydrous BPO compound without encapsulation in the base gel. BPO was not dissolved in ethanol or dimethyl sulfoxide (DMSO) as these do not simulate what would be used in an actual product. Each assay included a growth control without a test sample as a negative control. The test sample was placed at the bottom of a $50 \mathrm{~mL}$ tube, followed by $40 \mathrm{~mL}$ of either BHI $+3 \%$ HS or MRS broth. Overnight cultures of $G$. vaginalis were diluted to $10^{6} \mathrm{CFU} / \mathrm{mL}$ while the four Lactobacillus spp. were diluted to $10^{3} \mathrm{CFU} / \mathrm{mL}$. Cells were grown in direct contact with base gel, BPO gel, or BPO particles and incubated anaerobically at $37^{\circ} \mathrm{C}$. At $0-, 1^{-}, 3-, 6-$, 9-, 12- and 24-hour time intervals, $300 \mu \mathrm{L}$ of the culture was taken out for viable cell enumeration by the drop plate counting method as described by Herigstad et al. [26]. The experiment was carried out at least two times in duplicates. $Q$ test was performed and $Q_{90 \%}$ was set as rejection level.

2.6. Inhibition by BPO Released from the Gel. To avoid direct contact between the targeted cells and the released antimicrobial, the inhibition of $G$. vaginalis by the BPO gel through a $0.45 \mu \mathrm{m}$ diffusible membrane was tested via a control insert assay. Briefly, an overnight culture of $G$. vaginalis was diluted to $10^{6} \mathrm{CFU} / \mathrm{Ml}$; then $600 \mu \mathrm{L}$ of the culture dilution was transferred onto the bottom of a 24 -well control insert plate (Becton, Dickinson and Co., Bedford, MA, USA). The control inserts were then placed into the wells and $50 \mu \mathrm{L}$ of $1 \%$ BPO gel, base gel, or $0.5 \mathrm{mg}$ BPO particles was placed on 
TABLE 1: Zones of inhibition (mm) from well diffusion assay ${ }^{\mathrm{a}}$.

\begin{tabular}{lccccc}
\hline BP\% $(\mathrm{w} / \mathrm{w})$ & G. vaginalis & L. vaginalis & L. gasseri & L. plantarum & L. acidophilus \\
\hline 0 (base gel) & $0.00 \pm 0.00$ & $0.00 \pm 0.00$ & $0.00 \pm 0.00$ & $0.00 \pm 0.00$ & $0.00 \pm 0.00$ \\
0.01 & $0.50 \pm 0.00$ & $0.00 \pm 0.00$ & $0.00 \pm 0.00$ & $0.00 \pm 0.00$ & $0.00 \pm 0.00$ \\
0.10 & $0.67 \pm 0.26$ & $0.00 \pm 0.00$ & $0.00 \pm 0.00$ & $0.00 \pm 0.00$ & $0.00 \pm 0.00$ \\
0.25 & $1.08 \pm 0.20$ & $0.00 \pm 0.00$ & $0.00 \pm 0.00$ & $0.00 \pm 0.00$ & $0.00 \pm 0.00$ \\
0.50 & $1.17 \pm 0.26$ & $0.00 \pm 0.00$ & $0.00 \pm 0.00$ & $0.00 \pm 0.00$ & $0.00 \pm 0.00$ \\
1.00 & $1.67 \pm 0.26$ & $0.00 \pm 0.00$ & $0.00 \pm 0.00$ & $0.00 \pm 0.00$ & $0.00 \pm 0.00$ \\
2.50 & $1.92 \pm 0.38$ & $0.50 \pm 0.00$ & $0.50 \pm 0.00$ & $0.50 \pm 0.00$ & $0.50 \pm 0.00$ \\
5.00 & $2.25 \pm 0.42$ & $0.50 \pm 0.00$ & $0.50 \pm 0.00$ & $0.50 \pm 0.00$ & $0.50 \pm 0.26$ \\
7.50 & $2.42 \pm 0.38$ & $0.50 \pm 0.00$ & $0.50 \pm 0.00$ & $0.50 \pm 0.00$ & $0.58 \pm 0.20$ \\
10.00 & $2.58 \pm 0.38$ & $0.50 \pm 0.00$ & $0.75 \pm 0.27$ & $0.50 \pm 0.00$ & $0.67 \pm 0.26$ \\
Clindamycin $(100 \mu \mathrm{g} / \mathrm{mL})$ & $22.20 \pm 0.80$ & $17.30 \pm 0.50$ & $19.00 \pm 0.60$ & $21.80 \pm 0.80$ & $26.00 \pm 0.90$ \\
\hline
\end{tabular}

${ }^{a}$ The distance is measured from the edge of the loading well to the edge of the inhibition zone in millimeters using Vernier calipers. Experiments were conducted at least two times in triplicates. Mean values and their standard deviations are provided.

the top of each membrane. At the 0-, 1-, 3-, 6-, 9-, 12-, and 24hour time intervals, $200 \mu \mathrm{L}$ of culture was removed for enumeration by the drop plating method on HBT agar plates [26]. This experiment was carried out twice in duplicate for a total of 4 replicates. $Q$ test was performed and $Q_{90 \%}$ was set as rejection level.

2.7. Microbial Growth in $p H$ Adjusted Media. All cultures were grown in aforementioned, standard conditions for 24 hours at which time $200 \mu \mathrm{L}$ of overnight culture was transferred to $20 \mathrm{~mL}$ of $\mathrm{pH}$ adjusted media (MRS for Lactobacillus spp. and $\mathrm{BHI}+3 \% \mathrm{HS}$ for G. vaginalis). Media $\mathrm{pH}$ was adjusted to 4.5 (the average $\mathrm{pH}$ of all gels) through simple titration using either $0.1 \mathrm{M}$ hydrochloric acid or $30 \%$ lactic acid solution. Prior to adjustment, the $\mathrm{pH}$ of $\mathrm{BHI}+3 \% \mathrm{HS}$ is 7.05 while the $\mathrm{pH}$ of MRS is 6.16. Medium was filter-sterilized using $0.45 \mu \mathrm{m}$ filters (NALGENE, Rochester, NY, USA). Two hundred $\mu \mathrm{L}$ of culture was transferred into a sterile, 96well microplate (Corning, Inc., Corning, NY, USA). Wells containing bacteria in non-pH adjusted media and $\mathrm{pH}$ adjusted media without culture were used as controls. To prevent evaporation, $50 \mu \mathrm{L}$ of sterile mineral oil was pipetted gently on top of each well. Microplates were prepared anaerobically and turbidity was measured at $595 \mathrm{~nm}$ (Bio-Rad model 550 microplate reader, Bio-Rad Life Sciences, CA, USA) at 0, $1,3,6,9,12,18$, and 24 hours at $37^{\circ} \mathrm{C}$ in anaerobic conditions. This was performed twice in quadruplicate.

2.8. Rheological Measurements. Rheological evaluations of base gel and BPO gel formulations were measured using Hybrid Discovery HR-2 Rheometer (TA Instruments, New Castle, DE, USA) equipped with a $25 \mathrm{~mm}$ cross-hatched parallel steel plate and a temperature controlled parallel plate. The gap was maintained at $1.0 \mathrm{~mm}$ and the temperature control was set at $37^{\circ} \mathrm{C}$.

Oscillation frequency sweeps and oscillation shear stress sweeps were evaluated on the following formulations: base gel and 1\% BPO gel. In oscillation frequency sweeps, the shear stress was fixed at 10 pascal $(\mathrm{Pa})$, within the linear viscoelastic region, and $G^{\prime}$ and $G^{\prime \prime}$ were measured between 0.5 and
20 hertz $(\mathrm{Hz})$. In oscillation shear stress measurements, the frequency was fixed at $1 \mathrm{~Hz}$, and shear stress increased from 10 to $1000 \mathrm{~Pa}$.

Flow shear rate measurements at a variable shear rate from 0.1 to 1000 /second were conducted on the formulation. After gelation, the base gel and $1 \%$ BPO gel were diluted with VFS at $25 \%, 50 \%, 75 \%$, and $100 \%$ (gel/gel + VFS; v/v) and kept at $37^{\circ} \mathrm{C}$ in an incubator overnight prior to test. Viscosity using the flow shear measurements of diluted gel formulation was measured at a fixing frequency of $1 \mathrm{~Hz}$. At VSF of $25 \%$ the sample was briefly stirred prior to the measurement to provide with homogenous material. All experiments were conducted in triplicate.

2.9. Statistics and Figure Design. All statistical analyses were performed and figures in results section were graphed in Sigma Plot 11.0. The BPO structure and breakdown were made in MarvinSketch 5.12.1.

\section{Results and Discussion}

3.1. Influence of BPO Gel Formulations on Microbial Growth in Well Diffusion Assay. Well diffusion assays were performed to identify the lowest concentration of BPO (w/w) at which the BV-associated pathogen $G$. vaginalis could be inhibited. Mean values and associated standard deviation of the inhibition zones are shown in Table 1. Zones of inhibition were observed in G. vaginalis containing plates following exposure to $\mathrm{BPO}$ as low as $0.01 \%(\mathrm{w} / \mathrm{w})$. Zones were also confirmed in all tested higher concentrations. Zones of inhibition were observed at BPO concentrations of $2.5 \%$ or higher for all lactobacilli strains. No zones of inhibition were observed with the base gel, indicating no associated antimicrobial properties.

3.2. BPO Gel Formulation Selectively Inhibits G. vaginalis in Direct Contact Assay. The 1\% BPO gel was chosen for these tests as it represents the highest concentration that inhibited $G$. vaginalis but had no effect on the tested Lactobacillus spp. in the well diffusion assay (Figures 2(a)-2(e)). 


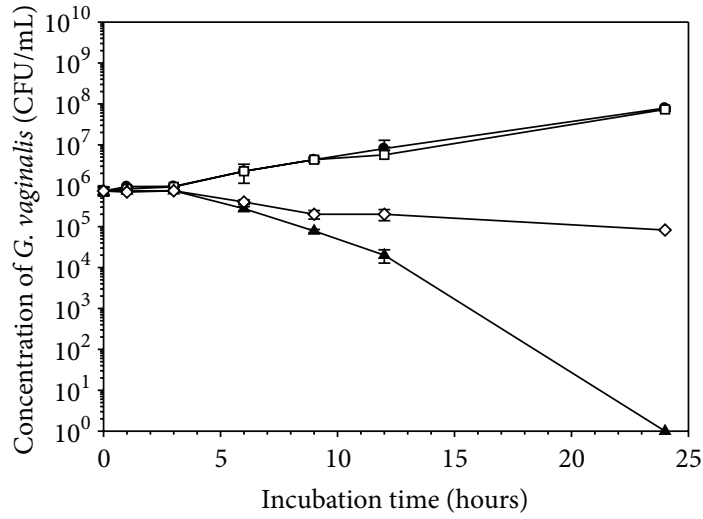

(a)

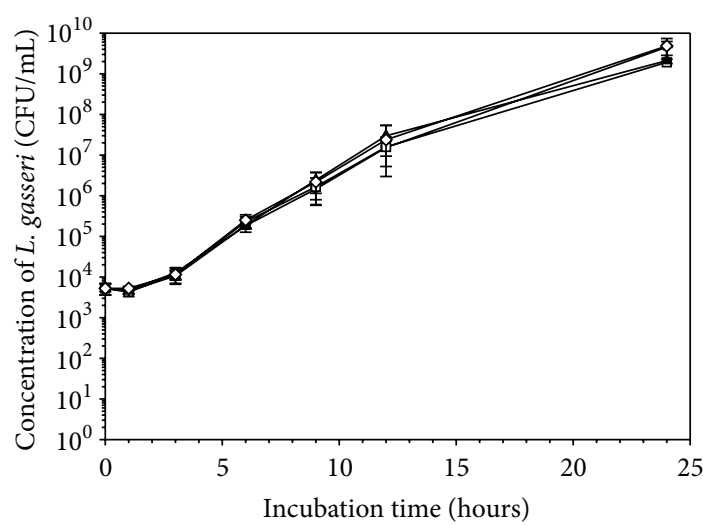

(c)

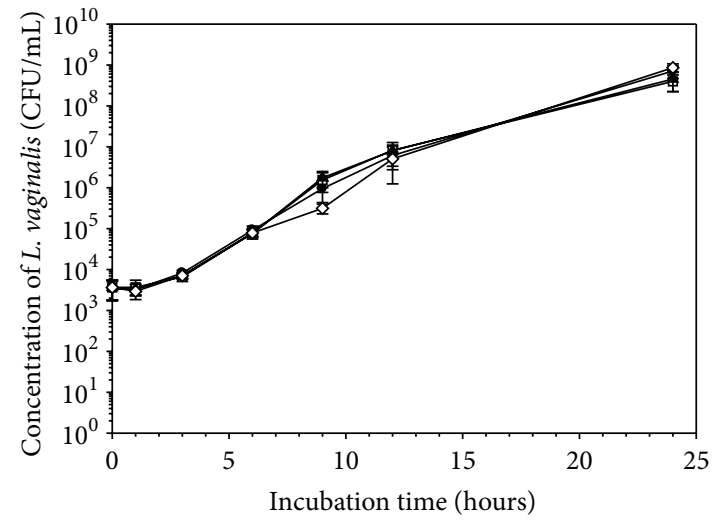

(b)

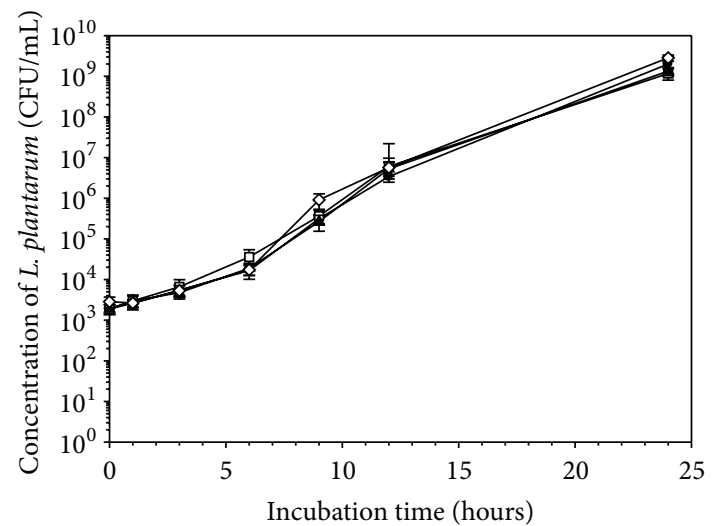

(d)

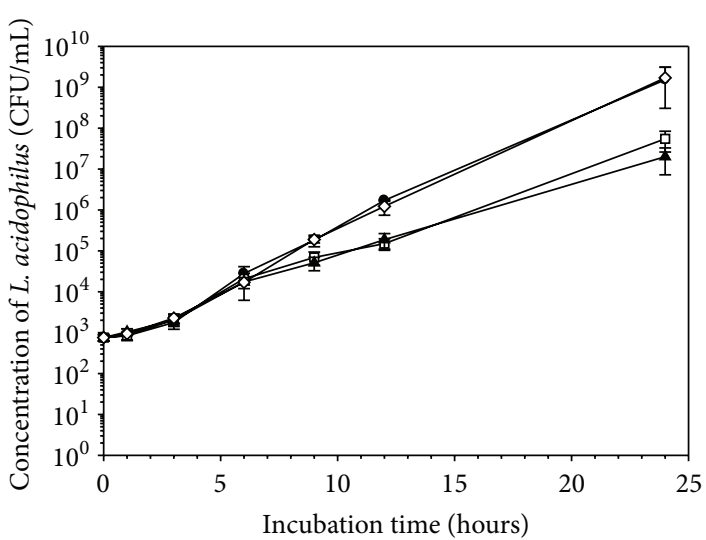

(e)

FIGURE 2: BPO inhibits G. vaginalis (a) but not healthy vaginal lactobacilli L. vaginalis (b), L. gasseri (c), L. plantarum (d), and L. acidophilus (e) in direct exposure experiments. $(\bullet)$ represents the negative control, $(\square)$ represents the base gel, $(\boldsymbol{\Delta})$ represents $1 \%$ BPO hydrogel, and $(\diamond)$ represents BPO particles. Experiments were conducted at least twice in duplicate. Mean values and standard deviations are shown.

To determine survivability of G. vaginalis, following 24 hours of direct exposure to $1 \% \mathrm{BPO}(\mathrm{w} / \mathrm{w})$, the drop plating technique was used. Following 24 hours of exposure, a six-log reduction of the viable $G$. vaginalis cells was observed when the microorganism was grown in contact with the $1 \%$ BPO gel. G. vaginalis cells were not inhibited by the base gel and grew up to $7.9 \times 10^{7} \mathrm{CFU} / \mathrm{mL}$ in the indirect inhibition assay or up to $7.3 \times 10^{7} \mathrm{CFU} / \mathrm{mL}$ in direct contact with the base gel. Therefore, we conclude that $1 \%$ BPO gel is effective in inhibiting G. vaginalis. By comparison, free BPO particles were less effective than the base gel, only reducing viable cell counts by approximately three logs (Figure 2(a)).

To determine the possible effect of direct contact with BPO on the viability of vaginal Lactobacillus spp., the direct contact assay was repeated under the same anaerobic conditions outlined for G. vaginalis. Following 24 hours of 


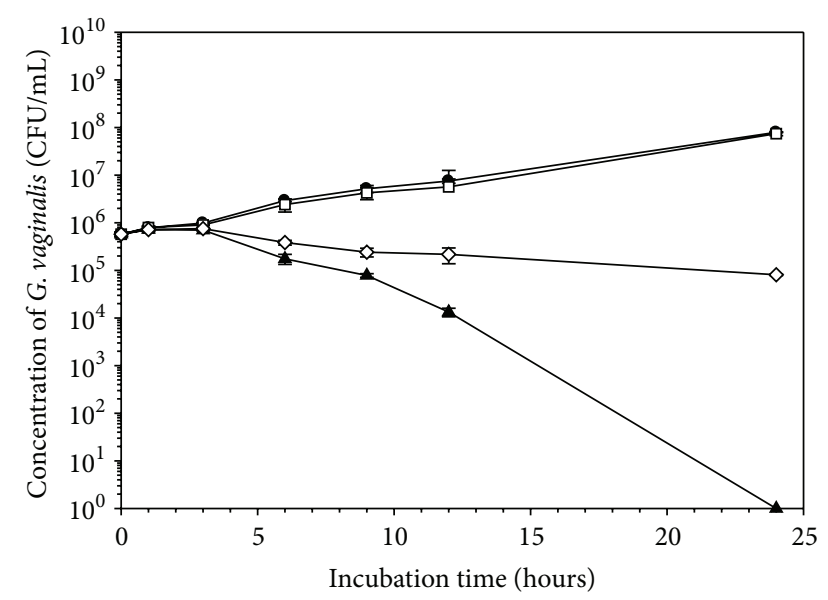

Figure 3: The effect of base gel, $1 \%$ BPO gel, and BPO particles on $G$. vaginalis in an indirect contact assay. All experiments were conducted twice in duplicate; $(\bullet)$ represents the negative control, ( $\square$ ) represents the base gel, $(\mathbf{\Delta})$ represents 1\% BPO hydrogel, and $(\diamond)$ represents BPO particles. Mean values and standard deviations are shown.

incubation, no significant inhibition was noted in direct contact assays in which cultures were grown in direct contact with BPO particles, the base gel, or the $1 \%$ BPO gel (Figures 2(b)-2(d)). A two-log reduction was noted in L. acidophilus cultures grown in contact with the $1 \% \mathrm{BPO}$ gel or the base gel (Figure 2(e)).

The 1\% BPO hydrogel formulation is capable of inhibiting the growth of the BV-associated pathogen G. vaginalis while having little to no impact on the growth of selected vaginal Lactobacillus spp.

Continued maintenance of the vaginal ecology may improve the rate of recovery from BV. As indicated by Mitchell et al., following antibiotic treatment, vaginal lactobacilli recovery is directly proportional to cure success rate [8]. Given that most antibiotics are nonselective in nature, it is imperative to consider the continued impact on the health of the individual by establishing methods that continually maintain a stable vaginal microbiome. The hydrogel described is capable of serving such a function. It is more selective, eliminating the tested pathogenic vaginal bacterium while supporting the growth of a healthy vaginal microbiome and reducing the recurrence rate of $\mathrm{BV}$ without supplementary lactobacilli treatment. The suggested and described approach is a technique that is a valuable preliminary assay that addresses the complex problem of BV. Further experiments based on our study will further elucidate the interaction within polymicrobial infections and help identify methodologies to further prevent inhibition of healthy microorganisms.

3.3. Effect of BPO on G. vaginalis Viability in an "Indirect Contact" Assay. Once the effect of the BPO formulations on the selected microorganisms was studied by the direct contact method, the effect in indirect exposure was evaluated using a control insert plate (Figure 3 ). The trials were conducted only with $G$. vaginalis at concentrations proven to be inhibitory
TABLE 2: Yield stress and cross-over of $G^{\prime}$ and $G^{\prime \prime}$ of tested formulations ${ }^{\mathrm{a}}$.

\begin{tabular}{lcc}
\hline Formulation & Yield stress $(\mathrm{Pa})$ & Cross-over of $G^{\prime}$ and $G^{\prime \prime}(\mathrm{Pa})$ \\
\hline Base gel & $32.42 \pm 1.73$ & $345.96 \pm 6.56$ \\
$1 \%$ BPO gel & $29.85 \pm 2.72$ & $332.58 \pm 12.01$ \\
\hline
\end{tabular}

${ }^{\mathrm{a}}$ Experiments were conducted in triplicate. Mean values and their standard deviations are shown.

to this vaginal pathogen because of the expense of the assay. Under conditions identical to those used in the indirect contact experiments, when exposed to gel-diffused BPO, G. vaginalis was inhibited similarly to what was observed in direct contact with 1\% BPO gel and BPO particles (a six-log reduction in viable cell count). In this, cells were placed at the bottom of the well while the effect of placing cells on top of the inserts was not assessed.

\subsection{Growth of Vaginal Microorganisms in pH Adjusted Media.} It was observed that in the "indirect contact" experiments the base gel did not influence $G$. vaginalis viability while BPO particles caused a $3-\log$ reduction in the number of viable cells. The base gel did however cause a 2-log reduction in L. acidophilus cultures. Therefore, one of the properties of the base gel (low $\mathrm{pH}$ of 4.5) was tested for possible effect on the growth of all tested microorganisms. L. plantarum, L. gasseri, and L. vaginalis grew normally while there was a slight inhibition of L. acidophilus and G. vaginalis, confirming that $\mathrm{pH}$ may play some role in reducing the bacterial viability (Figures 4(a)-4(e)).

3.5. Rheological Properties of Gel Formulations. The results of oscillation frequency are shown in Figure 5. In both tested formulations, from 0.5 to $20 \mathrm{~Hz}$, the storage modulus $\left(G^{\prime}\right)$ was always greater than loss modulus $\left(G^{\prime \prime}\right)$, indicating that this material exhibits "gel-like" properties. When frequency varied from 0.5 to $20 \mathrm{~Hz}, G^{\prime}$ of base gel was significantly reduced by $1 \%$ BPO $(P<0.01)$. When frequency varied from 1.2 to $20 \mathrm{~Hz}, G^{\prime \prime}$ of base gel was significantly reduced by $1 \%$ BPO $(P<0.05)$. But within measured range, $G^{\prime}$ of $1 \%$ BPO gel was above $600 \mathrm{~Pa}$, indicating that the formulation still possesses a solid-like behavior.

The results of oscillation shear stress are shown in Figure 6 and Table 2. Yield stress was defined as the shear stress at which its corresponding storage modulus was less than $95 \%$ of the average value from the first three detected storage moduli within the viscoelastic region, which represents initial gel elasticity. The yield stress of both formulations was approximately $30 \mathrm{~Pa}$, indicating that the gel deforms upon addition of stress causing it to shear thin.

The cross-over of $G^{\prime}$ and $G^{\prime \prime}$ represents breakdown of the gel microstructure allowing the material to flow as a viscous liquid. The cross-over of $G^{\prime}$ and $G^{\prime \prime}$ of both formulations was all above $300 \mathrm{~Pa}$, indicating that they possess a rigid microstructure [27].

The results of flow shear rate are shown in Figure 7. The viscosity of all tested formulations decreased with increasing shear rate, indicating a shear thinning behavior and their 


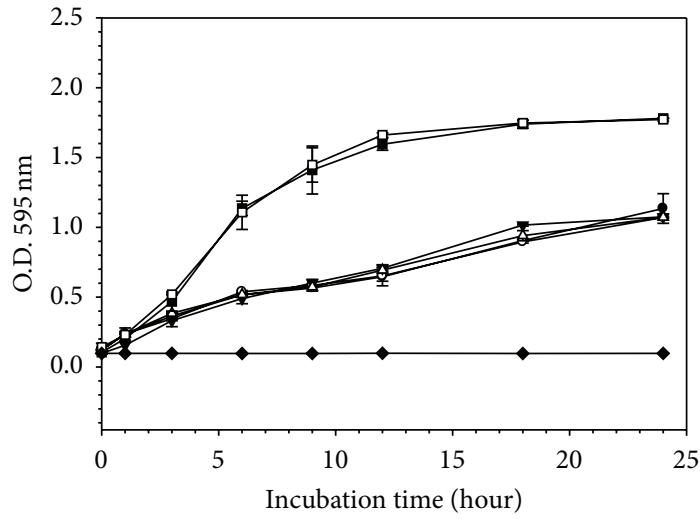

(a)

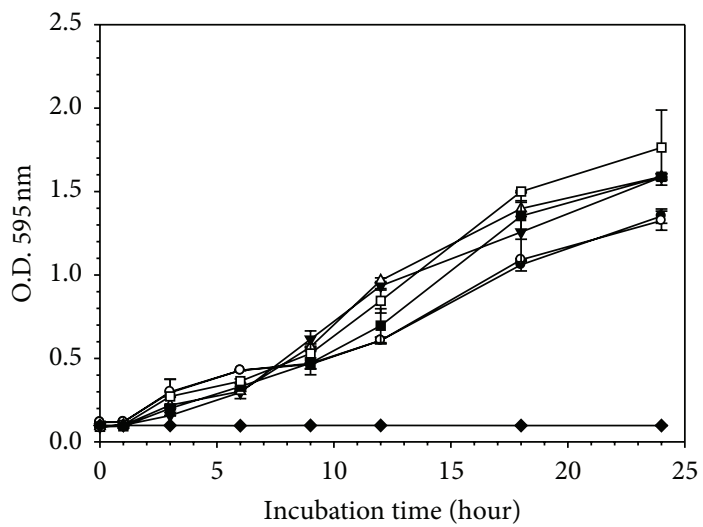

(c)

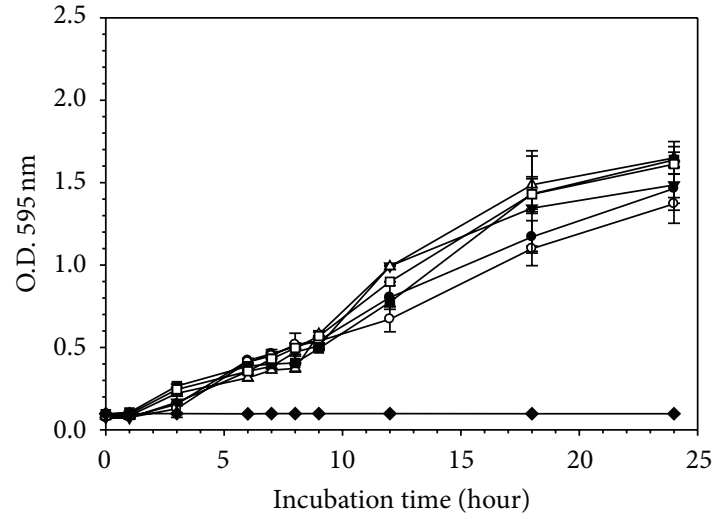

(b)

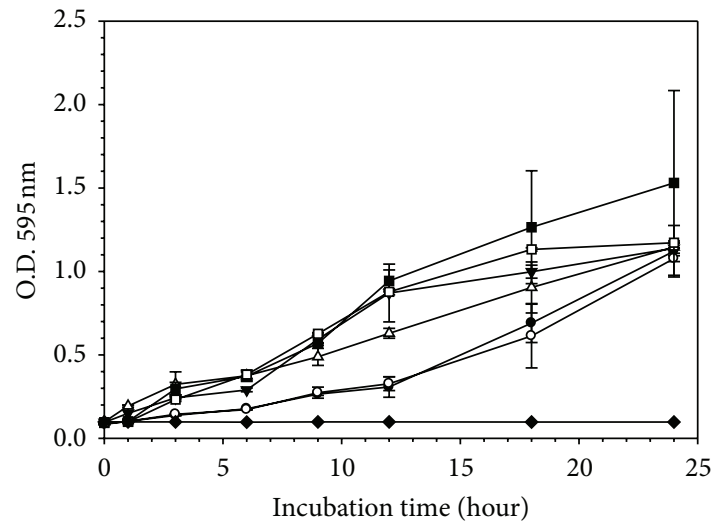

(d)

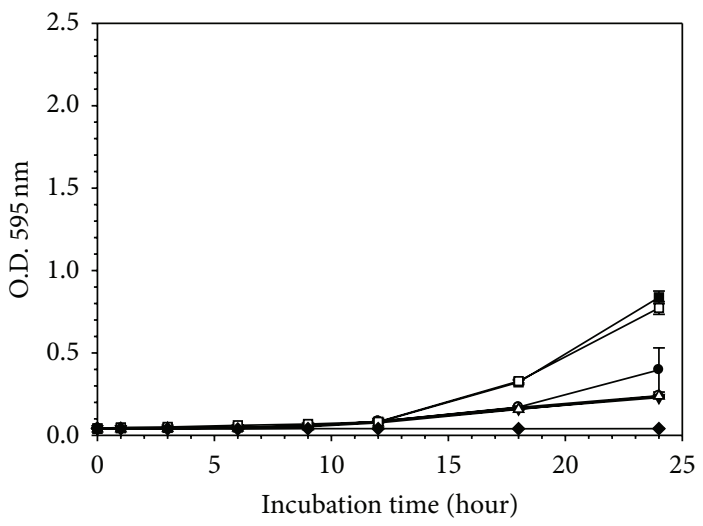

(e)

Figure 4: Influence of $\mathrm{pH}$ on growth of G. vaginalis (a), L. vaginalis (b), L. gasseri (c), L. plantarum (d), and L. acidophilus (e). Microbial growth was evaluated in media with $\mathrm{pH}$ altered to the average $\mathrm{pH}$ of the gels $(4.5)$ by $\mathrm{HCl}(\bullet, \circ)$, lactic acid $(\boldsymbol{\Lambda}, \Delta)$, and normal growth medium ( $\mathbf{\square}, \square)$ (MRS for lactobacilli spp. and BHI + 3\% HS for G. vaginalis). Sterile broth ( ) was also shown as negative control. Data were collected hourly (shown only $0,1,3,6,9,12,18$, and 24 h measurements). Experiments were conducted twice in quadruplicate.

injectability will be enhanced at higher shear rates. A high viscosity, at low shear rates, makes the formulations easier to stay along vagina mucosa [28]. The dispersion of BPO did not influence the viscosity of 50\%, 75\%, and $100 \%$ dilution of the base gel. However, $1 \%$ BPO greatly increased the viscosity of base gel when it was diluted with VFS at $25 \%$, which could be a result from the decrease in water component from
$1 \%$ BPO encapsulation. For both the base gel and the $1 \%$ $\mathrm{BPO}$ gel, the viscosity, as a function of shear rate ranging from 0.1 to $1000 / \mathrm{s}$, decreased when the formulation was diluted with VFS, indicating that the formulation will flow more easily when diluted with vaginal fluids in vivo. Viscosity significantly decreased upon dilution from $50 \%$ to $25 \%$, which may allow leakage to occur. Therefore, the presented intravaginal 


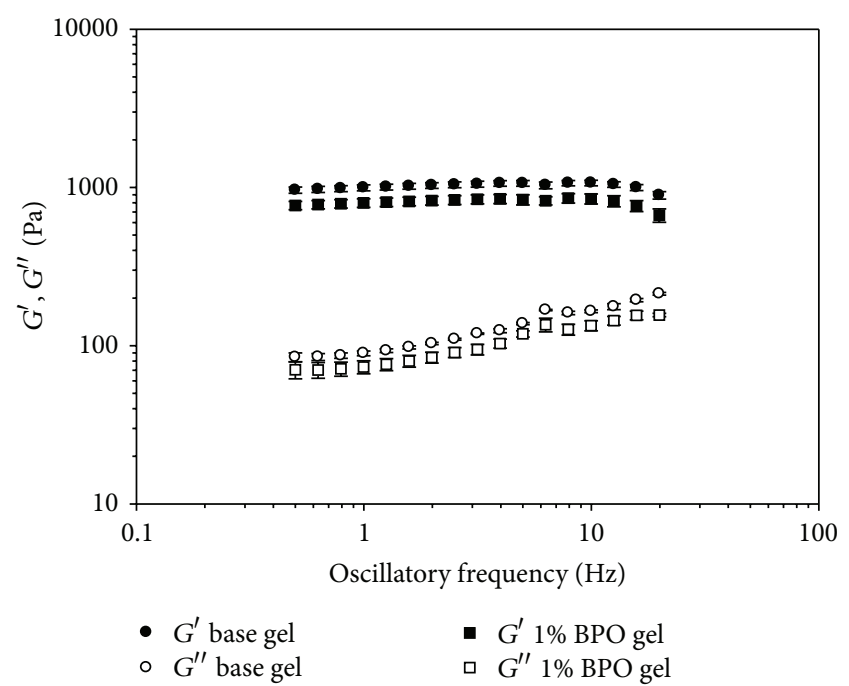

Figure 5: Storage modulus $\left(G^{\prime}\right)$ and loss modulus $\left(G^{\prime \prime}\right)$ as a function of oscillatory frequency $(\mathrm{Hz})$ on the base gel and $1 \%$ BPO gel. Experiment was conducted in triplicate. Mean values and standard deviations of three experiments are shown.

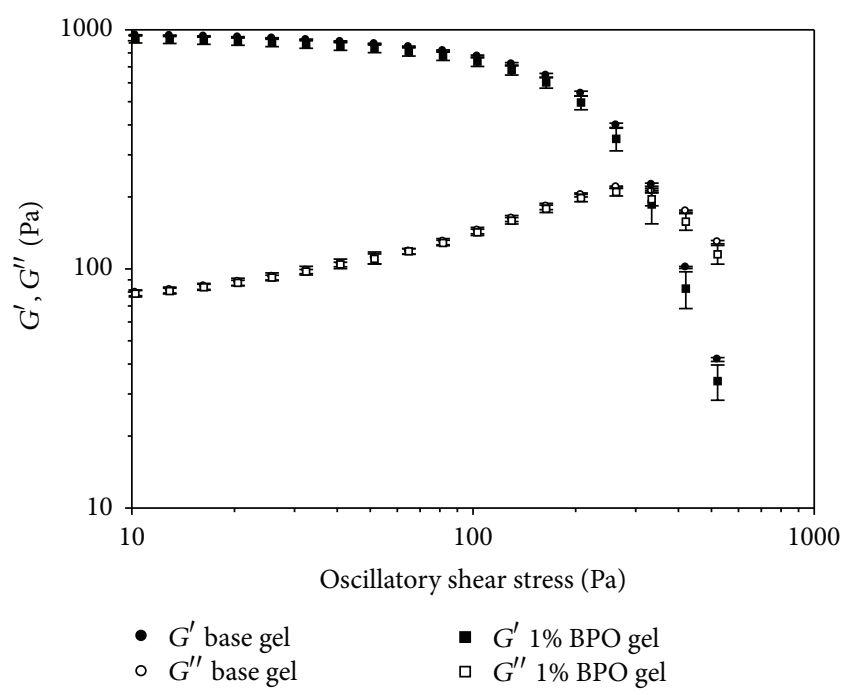

FIGURE 6: Storage modulus $\left(G^{\prime}\right)$ and loss modulus $\left(G^{\prime \prime}\right)$ as a function of oscillatory shear stress $(\mathrm{Pa})$ on the base gel and $1 \%$ BPO gel. Experiment was conducted in triplicate. Mean values and standard deviations of three experiments are shown.

drug delivery system presents multiple advantages over oral drug usage in its potential ability to control BV infection. On a much larger scale, the BPO-encapsulated hydrogel conceptually mimics the pathogen-inhibiting function of healthy peroxide-producing lactobacilli. This unique antibacterial profile supports its candidacy as a viable option for treatment and, perhaps equally importantly, for prevention of recurrence of BV. Prevention of the pathogen's recurrence is crucial for effective treatment and prophylaxis of bacterial vaginosis; this can be achieved with the gel's ability to sustain healthy microbiota, thus preventing the suppressed pathogens from dominating the environment. BPO has been used in flour bleaching and acne treatment for decades. It is permitted

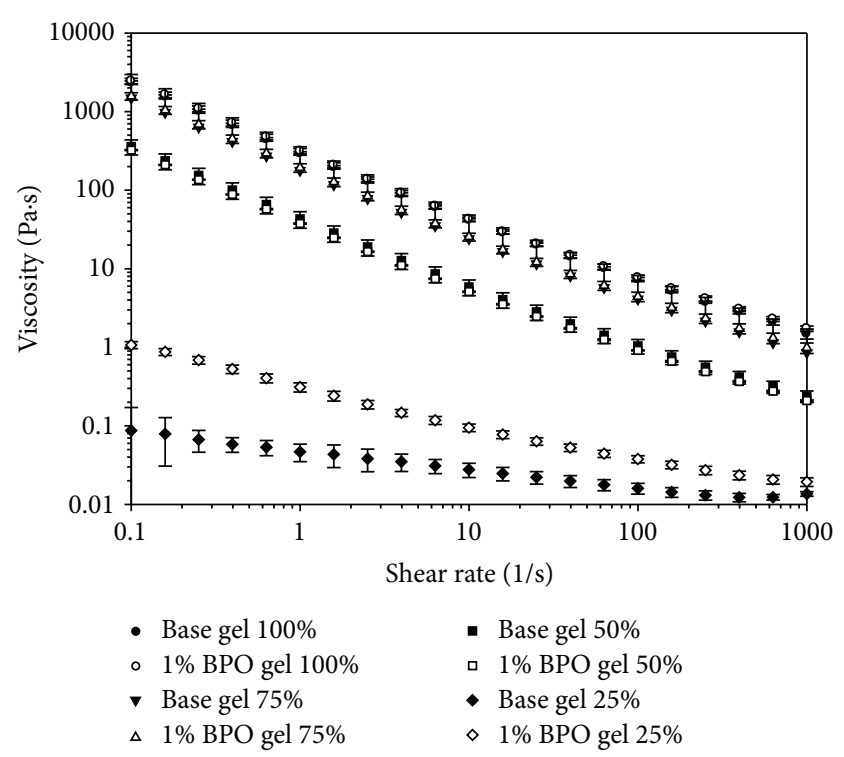

Figure 7: Viscosity (Pa.s) as a function of shear rate $(1 / \mathrm{s})$ of $100 \%$, $75 \%, 50 \%$, and $25 \%$ dilutions in VFS of base gel and $1 \%$ BPO gel. Experiment was conducted in triplicate. Mean values and standard deviations of three experiments are shown.

by the FDA for use as a topical drug up to $10 \%$ as active ingredient in product treatmenting acne, based on the studies proving the safety of BPO [29]. There is no evidence which suggests that the topical application of BPO gel or lotion is directly carcinogenic [30-32]. The danger of oxidative agents when used inappropriately [33] and isolated reports on possible carcinogenic effect of BPO in animal models [34] should not be neglected. However, the commonly accepted role played by the hydrogen peroxide-producing lactobacilli in controlling vaginal pathogens [9] inspires exploration for BPO use in vaginal health maintenance. Since most current studies focus on its safety when applied topically and exposed to ultraviolet light radiation, the safety of BPO when applied intravaginally should be assessed in other models before it is approved as a BV treatment.

Future studies will include gel delivery analysis in a mouse model and identification of possible irritation of vaginal epithelium at $1 \% \mathrm{BPO}$, which we propose as a possible dosage level.

\section{Conclusion}

In this study, we defined and evaluated a BPO-encapsulated hydrogel formulation capable of inhibiting the growth of the BV-associated pathogen $G$. vaginalis while having a limited effect on healthy lactobacilli in the vaginal ecosystem. The rheological properties of the gel show it to be suitable for the suggested application.

\section{Conflict of Interests}

The authors declare that there is no conflict of interests regarding the publication of this paper. 


\section{Authors' Contribution}

Shiqi Xu and Veronica L. Cavera equally contributed to this work.

\section{Acknowledgments}

The authors would like to thank the reviewers for their most valuable comments and suggestions which assisted in the overall improvement of this paper. The authors would also like to thank Mr. Joseph Cavera who gave considerable and concise editing advice.

\section{References}

[1] A. Swidsinski, H. Verstraelen, V. Loening-Baucke, S. Swidsinski, W. Mendling, and Z. Halwani, "Presence of a polymicrobial endometrial biofilm in patients with bacterial vaginosis," $\mathrm{PloS}$ ONE, vol. 8, no. 1, Article ID e53997, 2013.

[2] A. Swidsinski, W. Mendling, V. Loening-Baucke et al., "An adherent Gardnerella vaginalis biofilm persists on the vaginal epithelium after standard therapy with oral metronidazole," The American Journal of Obstetrics and Gynecology, vol. 198, no. 1, pp. el-e6, 2008.

[3] G. L. D. S. Santiago, I. Tency, H. Verstraelen et al., "Longitudinal qPCR study of the dynamics of L. crispatus, L. iners, A. vaginae, (sialidase positive) G. vaginalis, and P. bivia in the vagina," PloS ONE, vol. 7, no. 9, Article ID e45281, 2012.

[4] J.-P. Menard, F. Fenollar, M. Henry, F. Bretelle, and D. Raoult, "Molecular quantification of Gardnerella vaginalis and Atopobium vaginae loads to predict bacterial vaginosis," Clinical Infectious Diseases, vol. 47, no. 1, pp. 33-43, 2008.

[5] D. N. Fredricks, T. L. Fiedler, K. K. Thomas, B. B. Oakley, and J. M. Marrazzo, "Targeted PCR for detection of vaginal bacteria associated with bacterial vaginosis," Journal of Clinical Microbiology, vol. 45, no. 10, pp. 3270-3276, 2007.

[6] E. Biagi, B. Vitali, C. Pugliese, M. Candela, G. G. G. Donders, and P. Brigidi, "Quantitative variations in the vaginal bacterial population associated with asymptomatic infections: a realtime polymerase chain reaction study," European Journal of Clinical Microbiology and Infectious Diseases, vol. 28, no. 3, pp. 281-285, 2009.

[7] S. Srinivasan, N. G. Hoffman, M. T. Morgan et al., "Bacterial communities in women with bacterial vaginosis: high resolution phylogenetic analyses reveal relationships of microbiota to clinical criteria," PloS ONE, vol. 7, no. 6, Article ID e37818, 2012.

[8] C. Mitchell, L. E. Manhart, K. Thomas, T. Fiedler, D. N. Fredricks, and J. Marrazzo, "Behavioral predictors of colonization with Lactobacillus crispatus or Lactobacillus jensenii after treatment for bacterial vaginosis: a cohort study," Infectious Diseases in Obstetrics and Gynecology, vol. 2012, Article ID 706540, 6 pages, 2012.

[9] D. A. Eschenbach, P. R. Davick, B. L. Williams et al., "Prevalence of hydrogen peroxide-producing Lactobacillus species in normal women and women with bacterial vaginosis," Journal of Clinical Microbiology, vol. 27, no. 2, pp. 251-256, 1989.

[10] C. S. Bradshaw, L. A. Vodstrcil, J. S. Hocking et al., "Recurrence of bacterial vaginosis is significantly associated with posttreatment sexual activities and hormonal contraceptive use," Clinical Infectious Diseases, vol. 56, no. 6, pp. 777-785, 2013.

[11] S. R. Hymes, T. M. Randis, T. Y. Sun, and A. J. Ratner, "DNase inhibits Gardnerella vaginalis biofilms in vitro and in vivo,"
Journal of Infectious Diseases, vol. 207, no. 10, pp. 1494-1497, 2013.

[12] A. Aroutcheva, D. Gariti, M. Simon et al., "Defense factors of vaginal lactobacilli," The American Journal of Obstetrics and Gynecology, vol. 185, no. 2, pp. 375-379, 2001.

[13] E. Motevaseli, M. Shirzad, R. Raoofian et al., "Difference in vaginal lactobacilli composition of Iranian healthy and bacterial vaginosis infected women: a comparative analysis of their cytotoxic effects with commercial vaginal probiotics," Iranian Red Crescent Medical Journal, vol. 15, no. 3, pp. 199-206, 2013.

[14] M. Mart nez-Peña, G. Castro-Escarpulli, and M. G. AguileraArreola, "Lactobacillus species isolated from vaginal secretions of healthy and bacterial vaginosis-intermediate Mexican women: a prospective study," BMC Infectious Diseases, vol. 13, article 189, 2013.

[15] World Health Orgnization, "WHO model list of essential medicines adults 17th edition," 2011, http://www.who.int/ medicines/publications/essentialmedicines/en/index.html.

[16] N. K. Thakur, P. Bharti, S. Mahant, and R. Rao, "Formulation and characterization of benzoyl peroxide gellified emulsions," Scientia Pharmaceutica, vol. 80, no. 4, pp. 1045-1060, 2012.

[17] Y. El-Samragy, "Benzoyl peroxide chemical and technical assessment," Joint FAO/WHO Expert Committee on Food Additives, 61st meeting, 2004, http://www.fao.org/fileadmin/ templates/agns/pdf/jecfa/cta/63/Benzoylperoxide.pdf.

[18] K. Nozaki and P. D. Bartlett, "The kinetics of decomposition of benzoyl peroxide in solvents. I," Journal of the American Chemical Society, vol. 68, no. 9, pp. 1686-1692, 1946.

[19] J. das Neves and M. F. Bahia, "Gels as vaginal drug delivery systems," International Journal of Pharmaceutics, vol. 318, no. 12, pp. 1-14, 2006.

[20] S. Pendharkar, T. Magopane, P. G. Larsson et al., "Identification and characterisation of vaginal lactobacilli from South African women," BMC Infectious Diseases, vol. 13, article 43, 2013.

[21] E. Bilensoy, M. A. Rouf, I. Vural, M. Şen, and A. A. Hincal, "Mucoadhesive, thermosensitive, prolonged-release vaginal gel for clotrimazole: $\beta$-cyclodextrin complex," AAPS PharmSciTech, vol. 7, no. 2, article 38, pp. E54-E60, 2006.

[22] D. H. Owen, J. J. Peters, and D. F. Katz, "Rheological properties of contraceptive gels," Contraception, vol. 62, no. 6, pp. 321-326, 2000.

[23] D. H. Owen and D. F. Katz, "A vaginal fluid simulant," Contraception, vol. 59, no. 2, pp. 91-95, 1999.

[24] S. A. Waksman and H. C. Reilly, "Agar-streak method for assaying antibiotic substances," Industrial and Engineering Chemistry, vol. 17, no. 9, pp. 556-558, 1945.

[25] R. Liang, S. Xu, C. F. Shoemaker, Y. Li, F. Zhong, and Q. Huang, "Physical and antimicrobial properties of peppermint oil nanoemulsions," Journal of Agricultural and Food Chemistry, vol. 60, pp. 7548-7555, 2012.

[26] B. Herigstad, M. Hamilton, and J. Heersink, "How to optimize the drop plate method for enumerating bacteria," Journal of Microbiological Methods, vol. 44, no. 2, pp. 121-129, 2001.

[27] J.-Y. Kim, J.-Y. Song, E.-J. Lee, and S.-K. Park, "Rheological properties and microstructures of Carbopol gel network system," Colloid and Polymer Science, vol. 281, no. 7, pp. 614-623, 2003.

[28] J. das Neves, M. V. da Silva, M. P. Gonçalves, M. H. Amaral, and M. F. Bahia, "Rheological properties of vaginal hydrophilic polymer gels," Current Drug Delivery, vol. 6, no. 1, pp. 83-92, 2009. 
[29] U.S. Department of Health and Human Services, Food and Drug Administration and Center for Drug Evaluation and Research, "Guidance for industry: topical acne drug products for over-the counter human use: revision of labeling and clssification of benzoyl peroxide as safe and effective," http://www.fda.gov/downloads/Drugs/Guidances/ UCM259744.pdf.

[30] Consumer Healthcare Products Association, "Dermal oncogenicity study of benzoyl peroxide gels in rats," http://www.fda .gov/ohrms/dockets/dailys/02/Aug02/081302/8001e97b.pdf.

[31] C. M. Lerche, P. A. Philipsen, T. Poulsen, and H. C. Wulf, "Photocarcinogenesis and toxicity of benzoyl peroxide in hairless mice after simulated solar radiation," Experimental Dermatology, vol. 19, no. 4, pp. 381-386, 2010.

[32] J. H. Epstein, "Photocarcinogenesis promotion studies with benzoyl peroxide (BPO) and croton oil," Journal of Investigative Dermatology, vol. 91, no. 2, pp. 114-116, 1988.

[33] S. Papafragkou, A. Gasparyan, R. Batista, and P. Scott, "Treatment of portal venous gas embolism with hyperbaric oxygen after accidental ingestion of hydrogen peroxide: a case report and review of the literature," Journal of Emergency Medicine, vol. 43, no. 1, pp. e21-e23, 2012.

[34] T. J. Slaga, A. J. P. Klein-Szanto, L. L. Triplett, L. P. Yotti, and J. E. Trosko, "Skin tumor-promoting activity of benzoyl peroxide, a widely used free radical-generating compound," Science, vol. 213, no. 4511, pp. 1023-1025, 1981. 


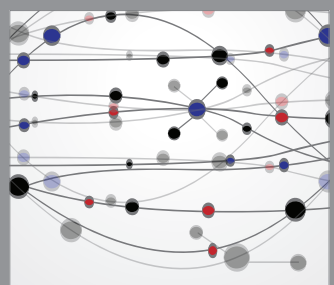

The Scientific World Journal
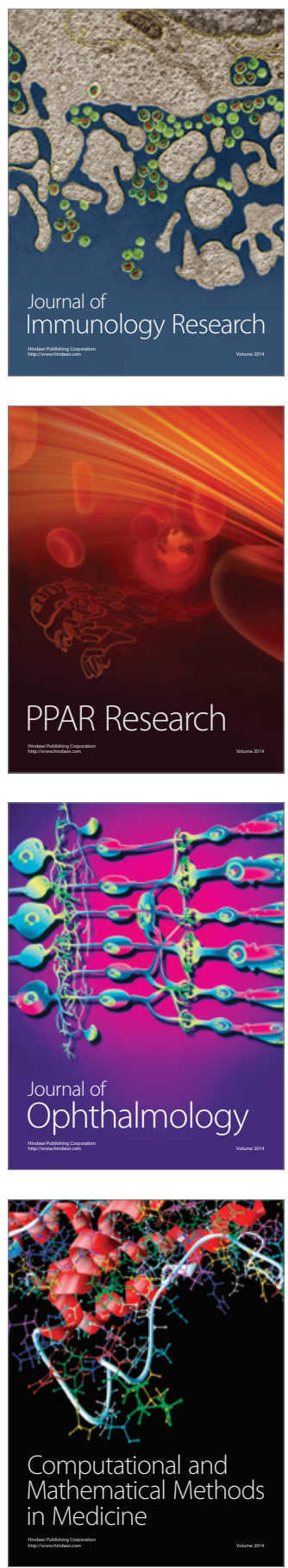

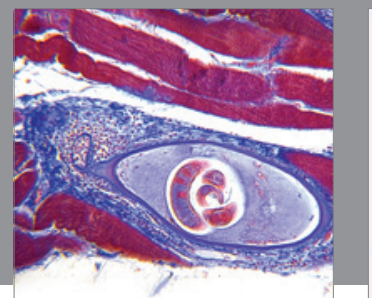

Gastroenterology

Research and Practice
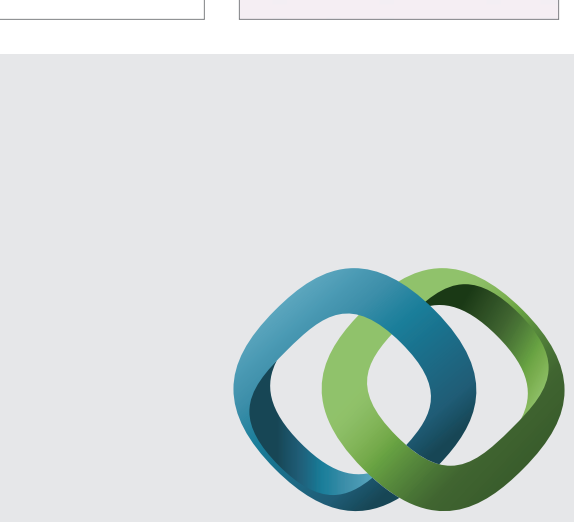

\section{Hindawi}

Submit your manuscripts at

http://www.hindawi.com
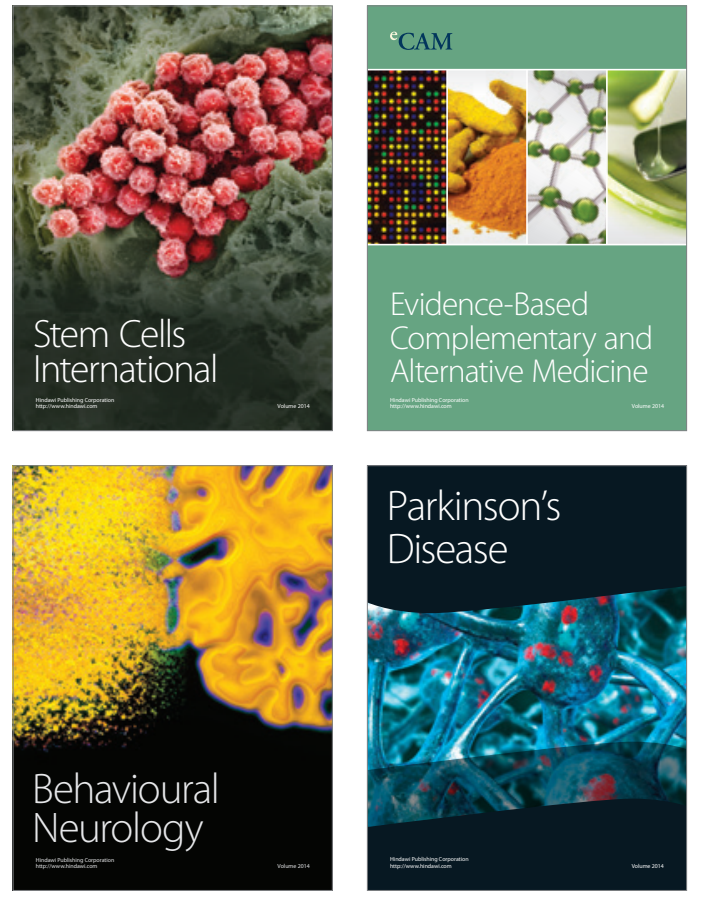
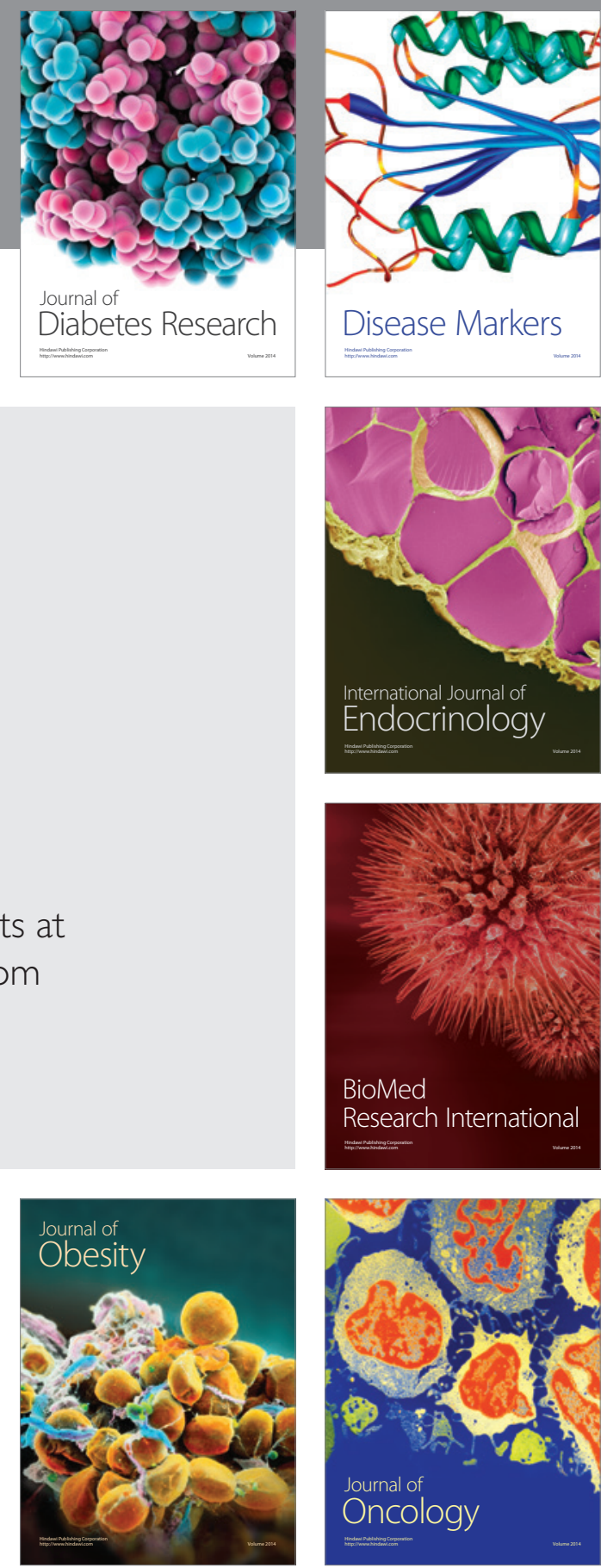

Disease Markers
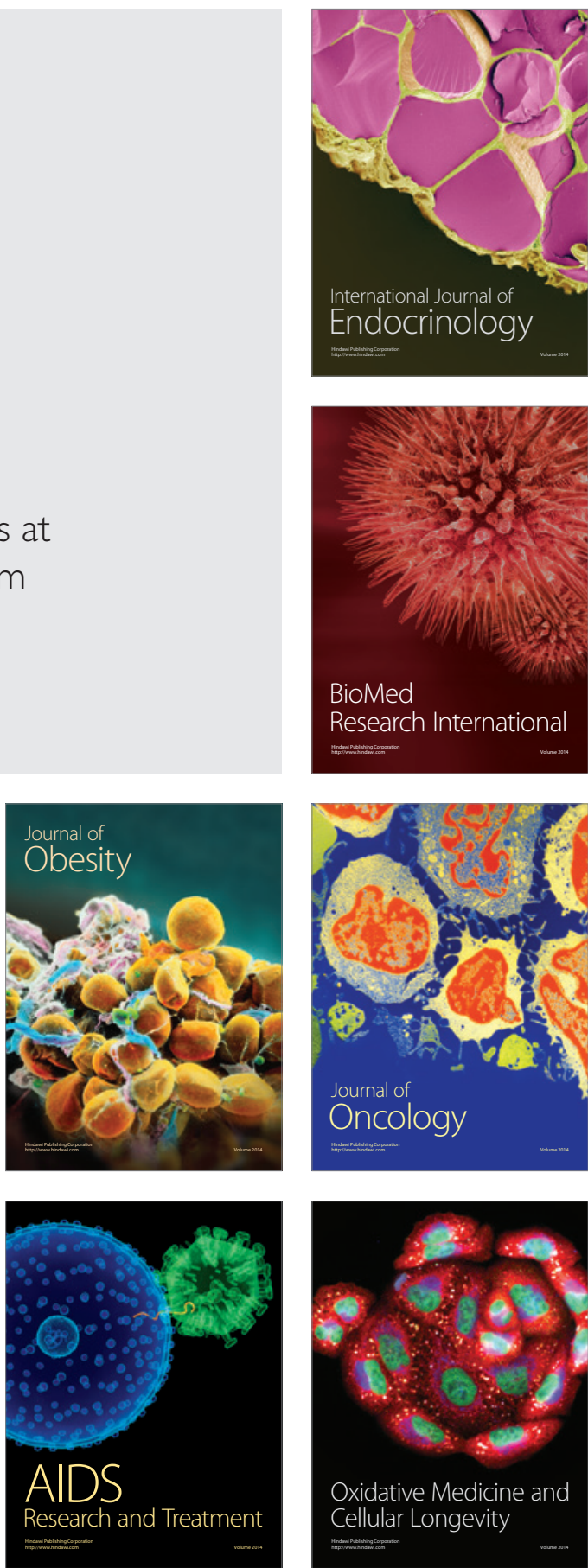